\title{
Area Efficient Design Analysis of Carry Look Ahead Adder
}

\author{
Anku Bala \\ M.E. Student \\ Department of Electronics and Communication \\ National Institute of Technical Teacher Training \\ And Research, Chandigarh
}

\author{
Rajesh Mehra \\ Associate Professor \\ Department of Electronics and Communication \\ National Institute of Technical Teacher Training \\ And Research, Chandigarh
}

\begin{abstract}
This paper provides a low power solution for Very Large Scale Integration. Power consumption of a circuit and its area occupied are major constraints for a VLSI designer. So in this paper we focused mainly on these two parameters by using different design methodologies such as Fully Automatic, Semi-custom and Fully Custom. Addition is basic operation in any system, but it requires more time as the number of bits are increased. So we use carry look ahead adder to reduce the processing time in circuits like processors or any other circuit. Design of CLA adder using semi-custom and fully custom approach can reduce the area and power consumption to a great extent.
\end{abstract}

\section{Keywords}

Carry Look Ahead Adder, VLSI, Automatic approach, fully custom approach.

\section{INTRODUCTION}

Fast addition is the arithmetic function for digital systems. It heavily impact the performance of digital systems [1]. Addition is also used to compute virtual physical address in fetching operation in all computers [2]. Many fast adders are available in market but the high speed with low power and less area is still challenging [3]. High performance circuits are continued to be a topic of interest. Addition circuits are fall under this group. The adders are need to be very fast because they are logic circuits designed to perform high speed arithmetic operations and are important block in digital systems because of their extensive use in all basic operations as subtraction, multiplication and division[4]. VLSI technology is a technology by which we can design complex system on chip where all elements of system like analog and digital circuit, passive element are integrated on single chip [5]. Therefore for a VLSI designer it is a major challenge to reduce the area of chip by using efficient optimization technique [5, 6]. Carry look ahead adder is proposed as an alternative for speed enhancements. Speed of Carry look ahead adder is usually determined by critical carry path delay and the carry critical path is data dependent and changes during the addition operation [7]. Carry look ahead adder is very basic building block for any digital circuit.

In VLSI, there are three domains structural domain, behavioral domain and physical domain. The structural domain deals with the schematic of transistors, gates, block diagram of circuit using flip-flops. In structural domain, the VLSI designer can design its circuit using transistors or gates. The paper is organized is as follows. The section I describes the overview of carry look ahead adder in which basic gate level diagram is described, section II describes the schematic of carry look ahead adder using transistors, section III describes the layout of carry look ahead adder which is obtained by VLSI tool. Simulation results are explained in section IV. Section V gives the conclusion of this paper [8].

There are three domains, structural domain, behavioral domain and physical domain. The structural domain deals with the schematic of transistors, gates, block diagram of circuits using flip-flops. In structural domain, the VLSI designer can design its circuit using transistors or gates by using wires. The behavioral domain deals with the front end technique in VHDL. The VLSI designer enters his design in form of equations. This domain cones under the front end design tool. Where designer does not know about what is going on at back end. The physical domain deals with the exact dimensions of the transistors. In this domain designer designs the transistors using rectangular strips of various layers. The physical domain is back end process.

\section{OVERVIEW OF CLA ADDER}

In ripple carry adder, the carry propagation time is the speed limiting factor. So if numbers of bits are increased then the delay in ripple carry adder is accordingly increased. So reducing the carry propagation is of great importance. Different design approaches have been employed to overcome the carry propagation. One widely used approach use principle of carry look ahead, which calculates the carry signal in advance. This type of adder is called carry look ahead adder [9]. Let A and B be the two four bit binary numbers and $\mathrm{S}$ and Cout being their sum and carry out respectively.

$$
\begin{aligned}
& \mathrm{A}=0101 \\
& \mathrm{~B}=0110 \\
& \mathrm{Sum}=1011 \text { and carry out }=0 \\
& P_{i}=A_{i} \oplus B_{i} \\
& G_{i}=A_{i} \cdot B_{i} \\
& \mathrm{~S}=\mathrm{P}_{\mathrm{i}} \oplus \mathrm{C}_{\mathrm{i}} \\
& C_{i+1}=\left(A_{i} \oplus B_{i}\right) \cdot C_{i}+A_{i} \cdot B_{i}
\end{aligned}
$$

Equation (1) shows the sum of two numbers A and B, eq (2) shows the product term of $\mathrm{A}$ and $\mathrm{B}$, eq (3) shows the final sum of the two binary bits and previous carry if any, eq (4) shows the final carry out signal which gives the result whether the final carry is generated or not. $P_{i}$ is known as carry propagate when $P_{i}$ is 1 the input carry is propagate to output 
carry. $G_{i}$ is known as carry generate signal because carry is generated when $G_{i}=1$.

$$
\begin{aligned}
& S_{0}=A_{0} \oplus B_{0} \oplus C_{0} \\
& S_{1}=A_{1} \oplus B_{1} \oplus C_{1} \\
& S_{2}=A_{2} \oplus B_{2} \oplus C_{2} \\
& S_{3}=A_{3} \oplus B_{3} \oplus C_{3}
\end{aligned}
$$

The above equations are used to calculate the sum of two 4 bit binary numbers. The XORing of two numbers A and B is done and the resultant is again XORed with initial carry to generate the Sum.

$$
\begin{aligned}
& C_{1}=\left(A_{0} \oplus B_{0}\right) C_{0}+A_{0} \cdot B_{0} \\
& C_{2}=\left(A_{1} \oplus B_{1}\right) C_{1}+A_{1} \cdot B_{1} \\
& C_{3}=\left(A_{2} \oplus B_{2}\right) C_{2}+A_{2} \cdot B_{2} \\
& C_{4}=\left(A_{3} \oplus B_{3}\right) C_{3}+A_{3} \cdot B_{3}
\end{aligned}
$$

The above equations show how the carry is calculated from previous carry and inputs bits. The four bit carry look ahead adder consists of 3 level of logic. First level generates all P and $G$ logic. The output signals from this level will be available after one propagation delay. In second level, the carry look ahead block will consists of four two level implementation logic. It generates carry signals and result will be available after three propagation delays. The third level includes the XOR gates which generate sum signals. The output of this signal will be available after four propagation delay [10]

\section{SCHEMATIC OF CLA ADDER}

The schematic of carry look ahead adder is designed in $\mathrm{DSCH}$. The schematic is designed using transistors or by logic gates. The first of all modules of gates are designed using transistors and then they are combined to form one complete module of one bit adder. In this design we have used three gates namely Exclusive OR(XOR) gate, OR gate and AND gate.

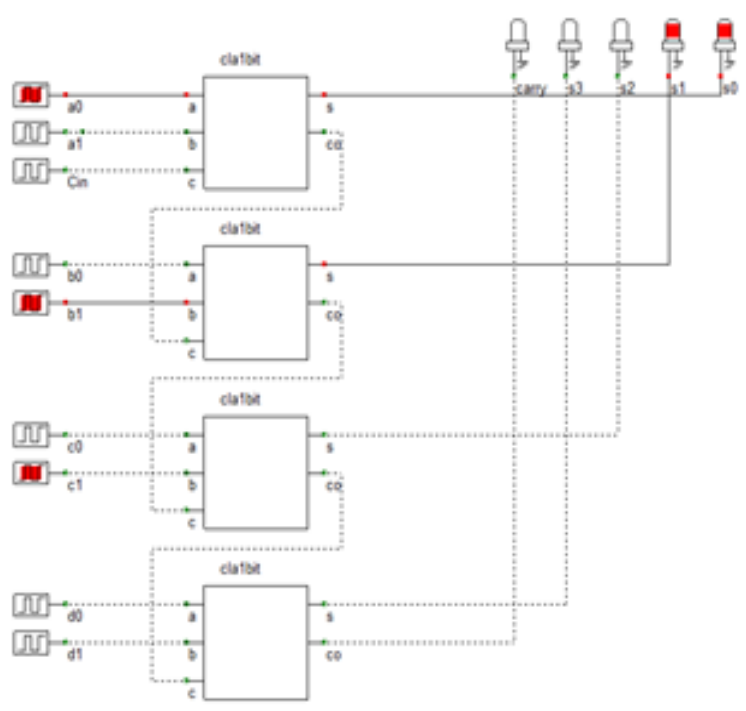

Fig 1 shows the block diagram of carry look ahead adder using modules created in DSCH tool. These modules contain the complete diagram for one bit addition. For four bit addition we connected four similar modules and made connections accordingly.

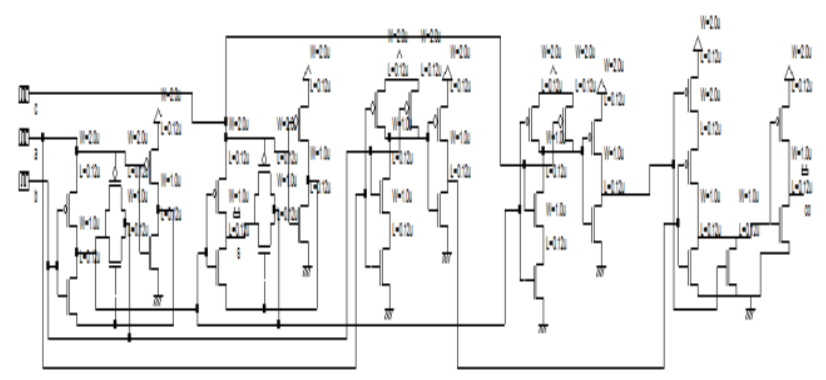

Fig 2: Transistor level schematic of one module

Fig 2 shows the internal schematic diagram of one module. The schematic consists of transistors.

\section{LAYOUT OF CLA ADDER}

In this paper we have used three approaches to design the carry look ahead adder and compared their results. The first approach is fully automatic. In fully automatic approach we first created the schematic in DSCH. DSCH is the Digital Schematic tool which is used to create a circuit diagram at gate or transistor level. After creating a design in DSCH tool, we have created a Verilog file and that Verilog file was compiled in micro-wind tool to auto-generate the layout. After this step auto-generated layout appeared on screen.

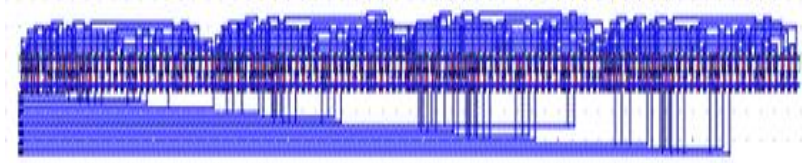

Fig 3: Layout of Fully Automatic design

Next step is semi-custom design. In this step we used only one VLSI tool i.e. micro-wind to create the layout directly without going to its schematic side. The layout was manually generated by us and its errors can be checked by DRC (Design Rule check).

Fig 1: Block diagram of CLA addder 


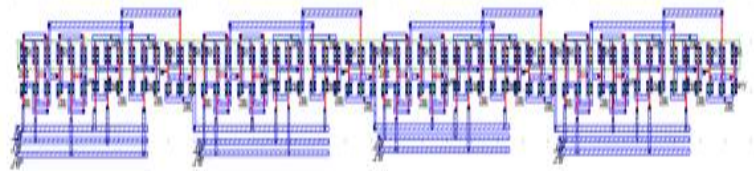

Fig 4: Layout of Semi-custom design
Fig 4 shows the layout of semi-custom approach which is obtained by creating the layout using transistors already available in the library. Connections are made using metal layers in layout.

There is one more approach called fully custom approach. In this we have started our design from scrap i.e. by first creating NMOS and PMOS transistors and then using these manually generated transistors to design gates and modules. When designing all the layout we have simulated the design and timing diagram is available with us. Fig 5 shows the Full custom design.

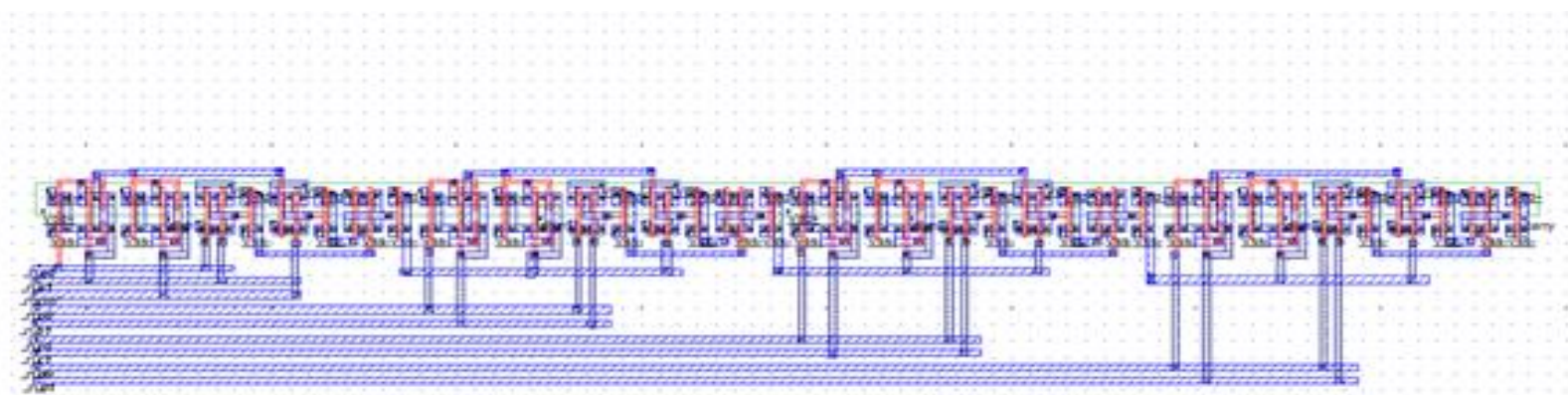

Fig 5: Layout of Full-custom design

\section{SIMULATION RESULTS}

Fig 6 shows the timing diagram of carry look ahead adder. This is the simulation result of schematic as shown in fig1. All the three designs have been simulated and result is as shown.

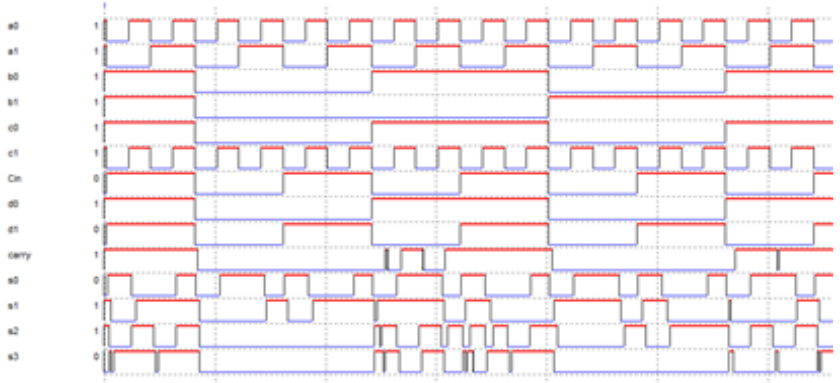

Fig 6: Timing Diagram of schematic of CLA adder

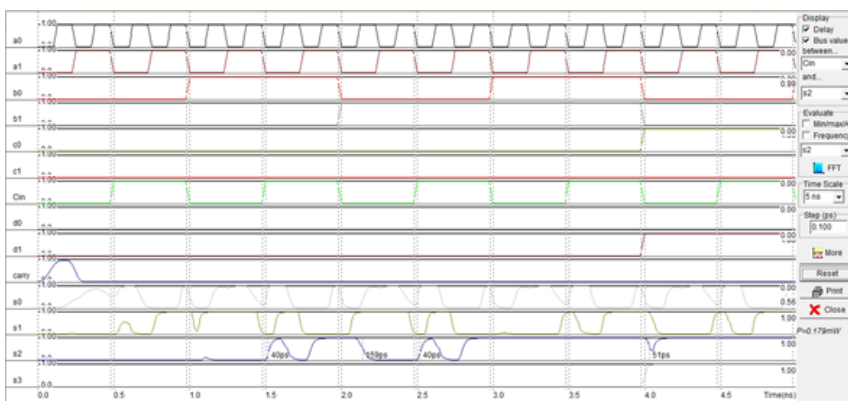

Fig 7: Simulation result of Fully Automatic design

Fig 7 shows the simulation results of fully automatic approach, and it has been shown that the power consumption is $179 \mu \mathrm{w}$.

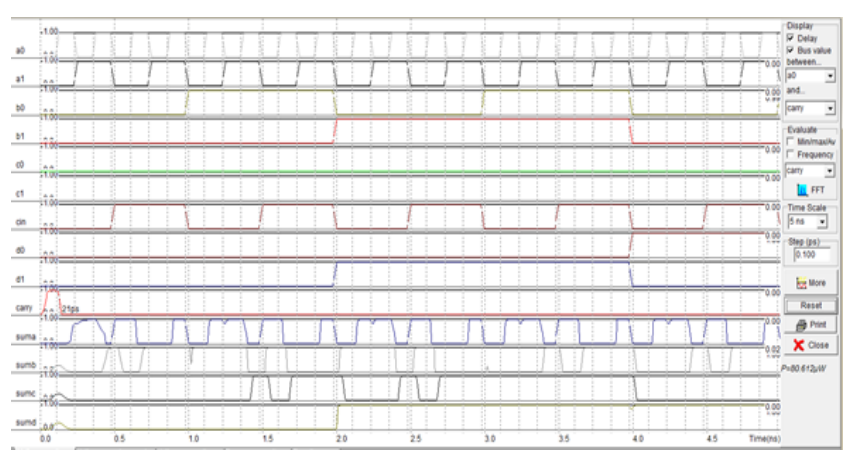

Fig 8: Simulation result of Semi-custom design

Fig 8 shows the simulation results of semicustom approach and the power consumption is $80.61 \mu \mathrm{w}$.

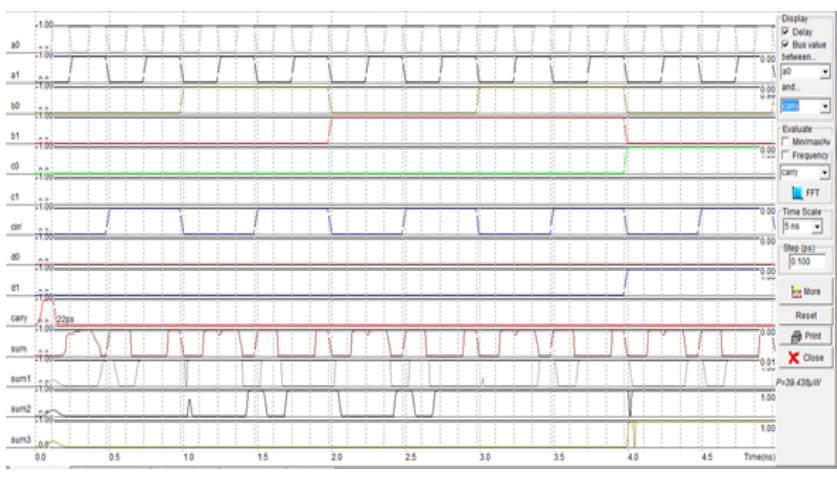

Fig 9: Simulation result of fully custom design

Fig 9 shows us the simulation results of fully custom design approach and power consumption of this design is $39.43 \mu \mathrm{w}$, which is least among the three. All the above simulation results are satisfying with the truth table of carry look ahead adder. 
Table 1: Comparison of design approaches

\begin{tabular}{|l|l|l|l|l|}
\hline $\begin{array}{l}\text { Para- } \\
\text { meters }\end{array}$ & Area & $\begin{array}{l}\text { Electrical } \\
\text { nodes }\end{array}$ & $\begin{array}{l}\text { Power } \\
\text { consumption } \\
(\mu w)\end{array}$ & $\begin{array}{l}\text { Current } \\
(m A)\end{array}$ \\
\hline $\begin{array}{l}\text { Fully } \\
\text { automatic }\end{array}$ & 1825.7 & 64 & 179 & 2.547 \\
\hline $\begin{array}{l}\text { Semi- } \\
\text { custom }\end{array}$ & 529.8 & 115 & 80.61 & 1.71 \\
\hline $\begin{array}{l}\text { Fully } \\
\text { custom }\end{array}$ & 269.3 & 127 & 39.43 & 0.887 \\
\hline
\end{tabular}

Table 1 shows the comparison of three design approaches with area and power. It can be seen that the power consumption is least in fully custom approach and same as the area.

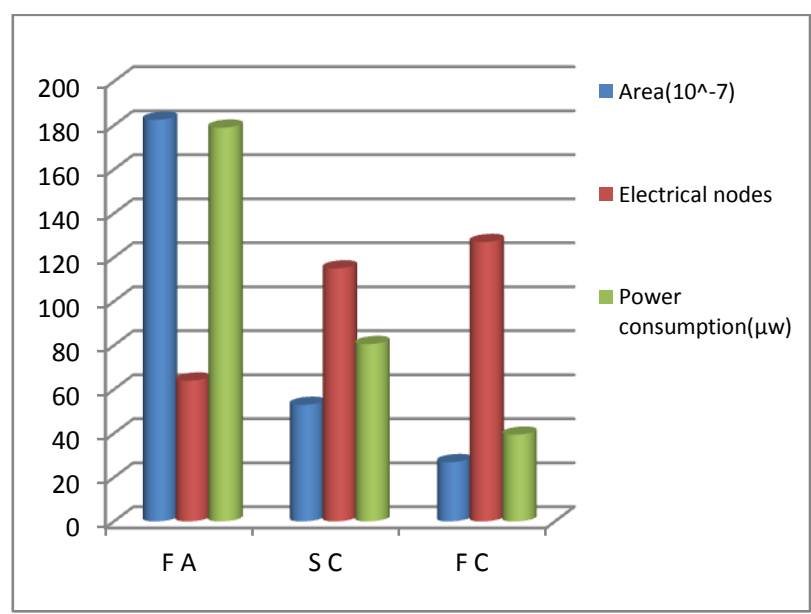

Fig 10: Comparison of various techniques

Fig 10 shows the graphical view of the comparison of all three approaches described above. FA stands for Full Automatic design, SC stands for semi-custom design and FC stands for fully custom design. It can be seen that power consumption and the area is greater in fully automatic approach, but as we decrease the area and power consumption the electrical nodes are automatically increased.

\section{CONCLUSION}

In this paper we have given a comparison of three design methods and concluded that the fully custom approach is best among the three where we can reduce the chip area and the power consumption as well. The semi-custom approach is moderate in which the area and power consumption is less than fully automatic but more than fully custom. In semi-custom design the area has been reduced up to $71 \%$ if we compare with fully automatic design and in fully custom the area has been reduced up to $85.2 \%$ as compare to fully automatic and $49 \%$ as compare to semi-custom design. As compare to fully automatic design the power consumption has been reduced up to $55 \%$ in case of semi-custom design and $77 \%$ in case of fully custom design.

\section{REFERENCES}

[1] C. Suba, S. Karthick, M. Prakash, "Analysis of Different Bit Carry Lookahead Adder with Reconfigurability in Low Power VLSI Using Verilog Code", International Journal of Innovative Research in Computer and Communication Engineering, ISSN (Print): 2320-9798, Vol. 2, Issue 11, November 2014, pp 6365-6371.

[2] Meena Aggarwal, Aastha Agarwal, Mr.Rajesh Mehra, "4-Input Decimal Adder Using $90 \mathrm{~nm}$ CMOS Technology", IOSR Journal of Engineering (IOSRJEN) e-ISSN: 2250-3021, p-ISSN: 2278-8719 Vol. 3, Issue 5(May. 2013), ||V4 || PP 48- 51.

[3] Laxmi Kumre, Ajay Somkuwar and Ganga Agnihotri, "Power Efficient Carry Propagate Adder", International Journal of VLSI design \& Communication Systems (VLSICS) Vol.4, No.3, June 2013, pp 125-134.

[4] Rajender Kumar, Sandeep Dahiya, “ Performance Analysis of Different Bit Carry Look Ahead Adder Using VHDL Environment", International Journal of Engineering Science and Innovative Technology (IJESIT) Volume 2, Issue 4, July 2013, pp 80-88

[5] Shilpa Thakur and Rajesh Mehra, "MOS Design And Single Supply Level Shifter Using 90nm Technology", Conference on Advances in Communication and Control Systems 2013 (CAC2S 2013), pp 150-153

[6] Dinesh Sharma and Rajesh Mehra, "Low Power, Delay Optimized Buffer Design using 70nm CMOS Technology", International Journal of Computer Applications (0975 - 8887) Volume 22- No.3, May 2011, pp 13-18

[7] Itamar Levi, Ori Bass, Asaf Kaizerman, Alexander Belenky and Alexander Fish, "High Speed Dual Mode Logic Carry Look Ahead Adder", IEEE International Symposium on Circuits and Systems (ISCAS), 2012 , pp $3037-3040$

[8] Jagannath Samanta, Mousam Halder, Bishnu Prasad De, "Performance Analysis of High Speed Low Power Carry Look-Ahead Adder Using Different Logic Styles", International Journal of Soft Computing and Engineering (IJSCE) ISSN: 2231-2307, Volume-2, Issue-6, Jan2013, pp 330-336

[9] Anjali Sharma Rajesh Mehra, "Area and Power Efficient CMOS Adder Design by Hybridizing PTL and GDI Technique", International Journal of Computer Applications (0975 - 8887) Volume 66- No.4, March 2013, pp 15-22.

[10] R.UMA,Vidya Vijayan, M. Mohanapriya, Sharon Paul, "Area, Delay and Power Comparison of Adder Topologies", International Journal of VLSI design \& Communication Systems (VLSICS) Vol.3, No.1, February 2012, pp 153-168. 